\title{
Re: Fosfomycin Trometamol in the Prophylaxis of Post-kidney Transplant Urinary Tract Infection: A Controlled, Randomized Clinical Trial
}

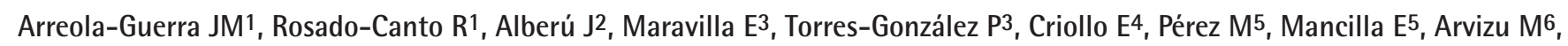
Morales-Buenrostro LE6, Vilatobá-Chapa M22, Sifuentes-Osornio J1

1IInstituto Nacional de Ciencias Médicas y Nutrición Salvador Zubirán, Department of Medicine, Tlalpan, México

2 Instituto Nacional de Ciencias Médicas y Nutrición Salvador Zubirán, Department of Transplantation, Tlalpan, México

3Instituto Nacional de Ciencias Médicas y Nutrición Salvador Zubirán, Laboratory of Clinical Microbiology, Tlalpan, México

4 Instituto Nacional de Ciencias Médicas y Nutrición Salvador Zubirán, Department of Pharmacy, Tlalpan, México

5Instituto Nacional de Cardiología Ignacio Chávez, Tlalpan, México

6Instituto Nacional de Ciencias Médicas y Nutrición Salvador Zubirán, Department of Nephrology-Mineral Metabolism, Tlalpan, México

Transpl Infect Dis 2018;20:e12980. doi: 10.1111/tid.12980. Epub 2018 Sep 10.

\section{EDITORIAL COMMENT}

This is a well designed randomized, controlled, double-blind clinical trial evaluating whether the addition of fosfomycin trometamol (FOS) to trimethoprim-sulfamethoxazole (TMP-SMX) prophylaxis following renal transplantation diminishes the risk of urinary tract infection (UTI) during the first 6 months following renal transplantation in adult patients. The intervention group (group 1, $n=32$ ) received FOS every 10 days and TMP-SMX three times per week; the control group (group $2, n=35$ ) received TMP-SMX daily. The authors found no difference in the time to first infection or the incidence of infections with the addition of FOS, and the study was halted at the time of intermediate analysis, as there was no possibility of benefit. A higher incidence of bacteriuria was reported than in previous years, with high levels of fosfomycin-resistant Klebsiella. Although interventions performed by the authors, such as catheter and stent placement, were quite standard, it is possible that these results may not be generalized to other settings with different patterns of infection and resistance like in patients undergoing urological procedures and in females with recurrent UTI in whom FOS has been superior.

Yarkın Kamil Yakupoğlu, MD

๑Copyright 2019 by the Association of Urological Surgery / Journal of Urological Surgery published by Galenos Publishing House. 